\title{
Primary ciliary dyskinesia with normal ultrastructure: three-dimensional tomography detects absence of DNAH11
}

\author{
Amelia Shoemark ${ }^{1,2}$, Thomas Burgoyne ${ }^{1}$, Robert Kwan ${ }^{1}$, Mellisa Dixon ${ }^{1}$, \\ Mitali P. Patel ${ }^{3}$, Andrew V. Rogers ${ }^{1}$, Alexandros Onoufriadis ${ }^{3}$, Juliet Scully ${ }^{3}$, \\ Farheen Daudvohra ${ }^{1}$, Thomas Cullup ${ }^{4}$, Michael R. Loebinger ${ }^{1}$, Robert Wilson ${ }^{1}$, \\ Eddie M.K. Chung ${ }^{3}$, Andrew Bush ${ }^{1,5}$, Hannah M. Mitchison ${ }^{3,6}$ and Claire Hogg ${ }^{1,5,6}$ \\ Affiliations: ${ }^{1}$ Royal Brompton and Harefield NHS Trust, London, UK. ${ }^{2}$ School of Medicine, University of \\ Dundee, Dundee, UK. ${ }^{3}$ Genetics and Genomic Medicine, University College London, UCL Great Ormond Street \\ Institute of Child Health, London, UK. ${ }^{4}$ North East Thames Regional Molecular Genetics Laboratory, Great \\ Ormond Street Hospital for Children NHS Foundation Trust, London, UK. ${ }^{5}$ Dept of Paediatrics, Imperial \\ College London, London, UK. ${ }^{6}$ Both authors contributed equally.
}

Correspondence: Amelia Shoemark, Dept of Paediatrics, Royal Brompton Hospital, London SW3 6NP, UK. E-mail: a.shoemarkarbht.nhs.uk

@ERSpublications

3D electron tomography identifies a defect in the cilia of patients with PCD and "normal ultrastructure" http://ow.ly/lZqd30hdPXM

Cite this article as: Shoemark A, Burgoyne T, Kwan R, et al. Primary ciliary dyskinesia with normal ultrastructure: three-dimensional tomography detects absence of DNAH11. Eur Respir J 2018; 51: 1701809 [https://doi.org/10.1183/13993003.01809-2017].

ABSTRACT In primary ciliary dyskinesia (PCD), motile ciliary dysfunction arises from ciliary defects usually confirmed by transmission electron microscopy (TEM). In 30\% of patients, such as those with DNAH11 mutations, apparently normal ultrastructure makes diagnosis difficult. Genetic analysis supports diagnosis, but may not identify definitive causal variants. Electron tomography, an extension of TEM, produces three-dimensional ultrastructural ciliary models with superior resolution to TEM. Our hypothesis is that tomography using existing patient samples will enable visualisation of DNAH11-associated ultrastructural defects. Dual axis tomograms from araldite-embedded nasal cilia were collected in 13 PCD patients with normal ultrastructure (DNAH11 n=7, HYDIN $\mathrm{n}=2$, CCDC65 $\mathrm{n}=3$ and DRC1 $\mathrm{n}=1$ ) and six healthy controls, then analysed using IMOD and Chimera software.

DNAH11 protein is localised to the proximal ciliary region. Within this region, electron tomography indicated a deficiency of $>25 \%$ of proximal outer dynein arm volume in all patients with DNAH11 mutations $(n=7)$ compared to other patients with PCD and normal ultrastructure $(n=6)$ and healthy controls (n=6). DNAH11 mutations cause a shared abnormality in ciliary ultrastructure previously undetectable by TEM. Advantageously, electron tomography can be used on existing diagnostic samples and establishes a structural abnormality where ultrastructural studies were previously normal. 


\section{Introduction}

Primary ciliary dyskinesia (PCD) is a genetic condition affecting $\sim 1$ in 15000 of the population. Ineffective mucociliary clearance, caused by disruption of ciliary motility, leads to the clinical phenotype of chronic sino-pulmonary disease [1]. Symptoms frequently start in the neonatal period with neonatal respiratory distress and include chronic nasal discharge and wet cough, progressing in childhood to recurrent upper and lower airway infections and eventual bronchiectasis [1]. Cilia are present in the embryonic node and defects in nodal cilia cause abnormalities of left-right laterality determination (situs) in $\sim 50 \%$ patients with PCD [1].

PCD is diagnosed using a combination of tests. These may include the measurement of nasal nitric oxide, analysis of cilia function by high-speed video microscopy and immunofluorescence analysis of protein expression [2, 3]. PCD diagnosis is ultimately confirmed by identification of a hallmark defect of ciliary ultrastructure visualised by transmission electron microscopy (TEM) or detection of nonambiguous biallelic mutations in a known PCD gene [2]. Normal ciliary ultrastructure is shown in figure 1. Patients with PCD most commonly have structural defects affecting the dynein arms and/or microtubular arrangement $[2,4,5]$. Apparently normal ultrastructure defined by conventional TEM is a common phenomenon and is thought to occur in up to $30 \%$ of cases [5-7]. Genotyping also leaves $25-50 \%$ cases unsolved [2]. In these cases, making a definitive diagnosis can be difficult.

Biallelic mutations in the DNAH11 gene are a common cause of PCD, accounting for $\geqslant 22 \%$ cases of PCD with "normal TEM" ultrastructure results $[8,9]$. The DNAH11 gene is thought to encode a heavy-chain dynein protein component of the axonemal outer dynein arm (ODA) present in the proximal part of the cilium (figure 1) [10]. Surprisingly, despite its sizeable molecular mass ( $520 \mathrm{kDa})$, the consequences of high-impact pathogenic mutations in DNAH11 predicted to be loss-of-function causing absence of the protein (e.g. nonsense, frameshift and essential splice site mutations) are undetectable by TEM [9]. This has long presented a diagnostic mystery, impeding definitive diagnosis, especially in cases where the identified DNAH11 mutations are not previously reported or known to be disease-producing. PCD is genetically heterogeneous and although some common causal variants are found, the majority of patients (including those carrying DNAH11 mutations) have unique mutations, some are additionally classed as variants of unknown significance by international clinical variant calling and reporting standards [11], e.g. amino acid substitution "missense" mutations of uncertain predicted pathogenicity [11]. DNAH11 mutations often confer a hyperfrequent ciliary beat visualised using high-speed video microscopy. However, this is not fully distinctive or diagnostic. Therefore, for patients with a DNAH11 mutation, due to normal ultrastructure and the presence of significant retained ciliary motility by light microscopy, a diagnosis of PCD can be missed and, furthermore, when a diagnosis is suspected it can be difficult to confirm using the existing structural or functional tests.

Dual axis electron tomography is an extension of the traditional TEM technique. It allows visualisation of ciliary structures in three dimensions (3D) enabling enhanced resolution and understanding of the ciliary ultrastructure $[12,13]$. We previously used electron tomography to demonstrate absence of the C2b central pair projection as the downstream structural defect of HYDIN mutations [14]. Electron tomography without averaging has been shown in one patient to have potential to detect disturbance of cilia ultrastructure arising from DNAH11 mutations [10].

In this study we tested the hypothesis that despite normal ultrastructure using standard TEM, 3D reconstruction and subtomographic averaging using existing patient samples might enable visualisation of DNAH11-associated ultrastructural defects. We investigated whether there is a loss of volume in $3 \mathrm{D}$ reconstructions of the ciliary ODA in DNAH11 PCD compared to healthy controls and PCD with normal ultrastructure not caused by DNAH11 mutations. In doing so, we aimed to investigate the potential of the 3D tomography technique for diagnosis and for advancing current knowledge of the effects on DNAH11 protein location of different DNAH11 mutations.

Support statement: H.M. Mitchison is supported by the Great Ormond Street Hospital Children's Charity, the UK PCD Family Support Group and the National Institute for Health Research (NIHR) Biomedical Research Centre at Great Ormond Street Hospital for Children NHS Foundation Trust and University College London. A. Shoemark is funded by a postdoctoral research fellowship from the NIHR and Health Education England (HEE-HCS-P13-04-004), mentored by C. Hogg, H.M. Mitchison and A. Bush. A. Bush was supported by the NIHR Respiratory Disease Biomedical Research Unit at the Royal Brompton and Harefield NHS Foundation Trust and Imperial College London. This report is independent research arising from a postdoctoral research fellowship supported by the NIHR and Health Education England. The views expressed in this publication are those of the authors and not necessarily those of the NHS, the NIHR or the Department of Health. Funding information for this article has been deposited with the Crossref Funder Registry.

Conflict of interest: None declared. 
a)

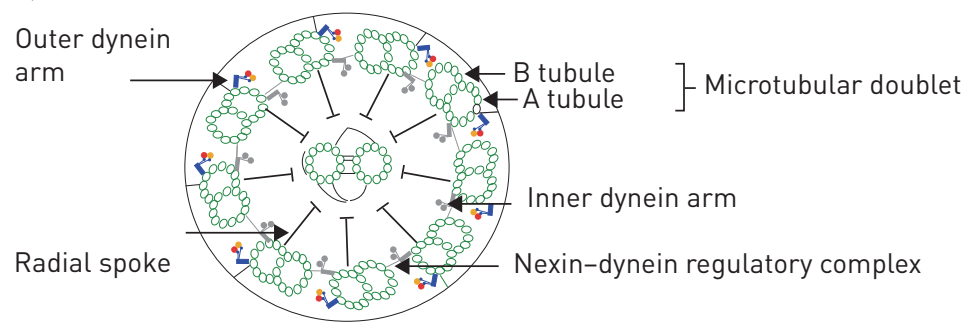

b)

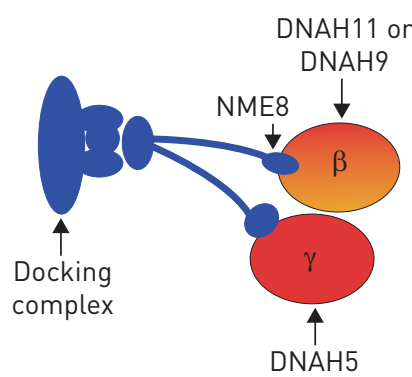

c)

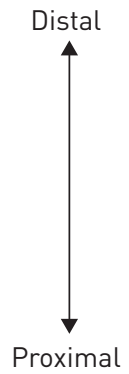

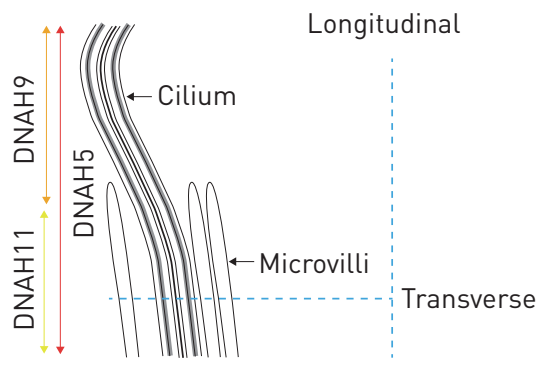

FIGURE 1 a) Schematic of 9+2 cilia axoneme ultrastructure in respiratory cells of a healthy individual, composed of nine peripheral microtubule doublets and a central microtubule pair. Each doublet contains an $\mathrm{A}$ microtubule and an incomplete B microtubule, with associated proteins attached to the A microtubule that include radial spokes and inner and outer dynein arms; nexin-dynein regulatory complexes link the peripheral doublets. The dynein arms contain heavy-chain dyneins, ATPases that hydrolyse ATP to provide energy for microtubule sliding and ciliary motility. One or more heavy chains form the "head" of the dynein arm which is attached to the dynein docking complex, which in turn is attached to the doublet A microtubule. b) The outer dynein arms contain two heavy chains primarily responsible for the motor action to drive ciliary movement, which are thought to be attached to the microtubules by a docking complex. Three different outer dynein axonemal heavy chains are recognised in human respiratory cilia: DNAH5, DNAH9 and DNAH11. c) In human nasal epithelial cell cilia, immunofluorescence studies show that DNAH5 localises along the whole axoneme, whereas DNAH9 localises only to the distal axoneme while DNAH11 occupies only the proximal axoneme (closer to the cell membrane and microvilli). Thus DNAH9 and DNAH11 act as the second heavy chain to DNAH5 in different regions.

\section{Methods}

Patient selection

Seven patients, aged 7-18 years, with known biallelic disease-causing mutations in DNAH11 were selected. Details of subjects and a summary of their diagnostic investigations are shown in table 1 . The study was approved by the London Bloomsbury research ethics committee under approval number 08/H0713/82.

Two comparator groups were used. Group one consisted of six healthy controls without PCD. Group two consisted of six subjects with a diagnosis of PCD and normal TEM, not carrying DNAH11 mutations. These cases were due to biallelic mutations in HYDIN $(\mathrm{n}=2)$, CCDC65 $(\mathrm{n}=3)$ and DRC1 $(\mathrm{n}=1)$. The PCD comparator group was chosen because ultrastructure is often considered normal in these cases, but it is not anticipated nor has it ever been seen that the ODA is affected by mutations in these three genes [1417]. These gene mutations are associated with isolated defects of the $\mathrm{c} 2 \mathrm{~b}$ central pair projection (HYDIN) and nexin-dynein regulatory complex (CCDC65 and DRC1). A subject with a loss-of-function mutation in DNAH5 with a known ODA defect by TEM was used as a negative control.

\section{Electron tomography sample preparation}

Ultrathin sections $(\sim 150 \mathrm{~nm})$ were cut using an ultra-microtome from blocks of nasal brushings embedded in araldite. Sections were stained with $2 \%$ uranyl acetate and lead citrate and subsequently labelled with $10 \mathrm{~nm}$ gold fiducial markers.

The following areas (plane of sections are shown in figure 1c) were assessed using tomography: 1) longitudinal sections of the axoneme at the proximal portion of the cilium; 2) longitudinal sections of the axoneme at the distal portion of the cilium; and 3) transverse cross-sections, at least six per field of view, at the proximal portion of the axoneme (in the region of the microvilli). 
TABLE 1 Clinical details and diagnostic test results, including genotype, of the subjects with primary ciliary dyskinesia due to mutations in DNAH11

\begin{tabular}{|c|c|c|c|c|c|c|c|c|}
\hline Patient & Sex & $\begin{array}{l}\text { Age at TEM } \\
\text { years }\end{array}$ & $\begin{array}{l}\text { Clinical signs and } \\
\text { symptoms }\end{array}$ & $\begin{array}{c}\mathrm{nNO} \\
\mathrm{nL} \cdot \mathrm{min}^{-1} \\
\text { (normal } \\
>77 \mathrm{~nL} \cdot \mathrm{min}^{-1} \text { ) }\end{array}$ & $\begin{array}{c}\text { Light microscopy } \\
\text { HSVM } \\
\text { CBF Hz }\end{array}$ & $\begin{array}{c}\text { TEM } \\
\text { DA defects } \\
\text { MTD } \\
\text { defects }\end{array}$ & DNAH11 mutations & $\begin{array}{l}\text { Genotype and predicted } \\
\text { protein effect }\end{array}$ \\
\hline \#1 & Male & 18 & $\begin{array}{l}\text { Recurrent chest } \\
\text { infections/rhinitis }\end{array}$ & 29 & $\begin{array}{r}\text { Static } \\
0 \mathrm{~Hz}\end{array}$ & $\begin{array}{l}\text { Normal } \\
\text { DA 0\% } \\
\text { MTD } 4 \%\end{array}$ & $\begin{array}{l}\text { [nonsense](;) } \\
\text { [frameshift] }\end{array}$ & $\begin{array}{c}\text { [c.3727G>T]; } \\
\text { [c.13531_13532insTTCAGGCTGAAGA] } \\
\text { [p.Glu1243*]; [p.Ala4511Valfs*13] }\end{array}$ \\
\hline \#2 & Female & 15 & $\begin{array}{l}\text { Recurrent chest } \\
\text { infections/rhinitis }\end{array}$ & 6 & $\begin{array}{c}\text { Static and } \\
\text { hyperfrequent } \\
22.6 \mathrm{~Hz}\end{array}$ & $\begin{array}{l}\text { Normal } \\
\text { DA 0\% } \\
\text { MTD 5\% }\end{array}$ & $\begin{array}{l}\text { [missense](;) } \\
\text { [frameshift] }\end{array}$ & $\begin{array}{c}{[c .7663 \mathrm{C}>\mathrm{T}] ;[\text { [c.4410_4413del] }} \\
{[\text { p.Gln2555*]; [p.Ser1470Argfs*6] }}\end{array}$ \\
\hline \#3 & Female & 14 & $\begin{array}{l}\text { Situs inversus + } \\
\text { recurrent chest infection }\end{array}$ & 14 & $\begin{array}{c}\text { Static and } \\
\text { hyperfrequent } \\
12.8 \mathrm{~Hz}\end{array}$ & $\begin{array}{l}\text { Normal } \\
\text { DA 0\% } \\
\text { MTD } 7 \%\end{array}$ & $\begin{array}{l}\text { [nonsense] }(;) \\
\text { [nonsense] }\end{array}$ & $\begin{array}{c}{[c .5506 \mathrm{C}>\mathrm{T}] ;[\text { c.5636T>A] }} \\
{[\text { p.Arg1836*]; [p.Leu1879*] }}\end{array}$ \\
\hline$\# 4$ & Female & 7 & $\begin{array}{l}\text { Recurrent chest } \\
\text { infections/rhinitis }\end{array}$ & 14 & $\begin{array}{c}\text { Slow with reduced } \\
\text { bending } \\
6.15 \mathrm{~Hz}\end{array}$ & $\begin{array}{l}\text { Normal } \\
\text { DA 3\% } \\
\text { MTD 9\% }\end{array}$ & $\begin{array}{l}\text { [frameshift](;) } \\
\text { [frameshift] }\end{array}$ & $\begin{array}{c}\text { [c.2832dup]; [c.13240dup] } \\
\text { [p.Gln945Serfs*10]; [p.Thr4414Asnfs*34] }\end{array}$ \\
\hline \#5 & Female & 10 & $\begin{array}{l}\text { Recurrent chest } \\
\text { infections/rhinitis }\end{array}$ & 50 & $\begin{array}{l}\text { Static and } \\
\text { hyperfrequent } \\
\text { (ND) }\end{array}$ & $\begin{array}{l}\text { Normal } \\
\text { DA 0\% } \\
\text { MTD 3\% }\end{array}$ & $\begin{array}{l}\text { [missense](;) } \\
\text { [missense] }\end{array}$ & $\begin{array}{c}{[c .6506 \mathrm{C}>T] ;[c .6506 \mathrm{C}>\mathrm{T}]} \\
{[p . \text { Ser2169Leu]; [p.Ser2169Leu] }}\end{array}$ \\
\hline \#6 & Female & 14 & $\begin{array}{l}\text { Situs inversus + } \\
\text { recurrent chest infection }\end{array}$ & 20 & $\begin{array}{c}\text { Static and } \\
\text { hyperfrequent } \\
13.3 \mathrm{~Hz}\end{array}$ & $\begin{array}{l}\text { Normal } \\
\text { DA } 0 \% \\
\text { MTD } 4 \%\end{array}$ & $\begin{array}{l}\text { [nonsense](;) } \\
\text { [nonsense] }\end{array}$ & $\begin{array}{l}\text { [c.3220G>T]; [c.13090C>T] } \\
\text { [p.Glu1074*]; [p.Arg4364*] }\end{array}$ \\
\hline \#7 & Male & 9 & $\begin{array}{l}\text { Recurrent chest } \\
\text { infections/rhinitis }\end{array}$ & 8 & $\begin{array}{l}\text { Hyperfrequent } \\
19.6 \mathrm{~Hz}\end{array}$ & $\begin{array}{l}\text { Normal } \\
\text { DA 0\% } \\
\text { MTD } 19 \%\end{array}$ & $\begin{array}{l}\text { [nonsense](;) } \\
\text { [splice site] }\end{array}$ & $\begin{array}{l}{[c .3544 \mathrm{C}>\mathrm{T}]} \\
{[\text { [c.8798-5G>A] }} \\
{\left[\mathrm{p} . \operatorname{Arg} 1182^{*}\right]}\end{array}$ \\
\hline
\end{tabular}

TEM: transmission electron microscopy; nNO: nasal nitric oxide; HSVM: high-speed video microscopy; CBF: ciliary beat frequency; DA: dynein arm; MTD: microtubular doublet; ND: not done, because dyskinesia frequency measurements were not taken. ${ }^{\#}$ : c.13531_13532insTTCAGGCTGAAGA mutation (patient 1) has not been proven for pathogenicity. 
Generation of electron tomograms

A JEOL 1400+ TEM (Welwyn Garden City, UK) was used to collect a series of images at different stage tilts. $4 \mathrm{~K} \times 4 \mathrm{~K}$ digital images at $\times 20000$ magnification were captured using JEOL tomography software and a digital camera (AMT 16X, Deben, Bury St Edmunds, UK). The software automatically adjusts stage position, focus and exposure. The specimen was tilted through angles of $+65^{\circ}$ to $-65^{\circ}$ and images acquired at increments of $1^{\circ}$. Following image generation the sample was manually rotated $90^{\circ}$ and in the same region a second series of images was acquired. The two image series were aligned and combined to produce a dual axis reconstruction using IMOD software [18, 19].

\section{Subtomographic averaging}

Subtomographic averaging was conducted to enhance the ODA structure using particle estimation for electron tomography, part of the IMOD software package [19]. The regions chosen for averaging of longitudinal tomograms were at 96-nm intervals [20]. For transverse cross-sections the centre of the A microtubule of each microtubule doublet (figure 1) was selected as the centre for averaging. For quality control, averages in which the inner dynein arm and radial spokes could be visualised were considered acceptable for further analysis.

\section{Tomography data analysis}

Imaging, modelling and quantification of the ODAs was performed using the UCSF Chimera programme developed by the Resource for Biocomputing, Visualisation, and Informatics at the University of California San Fransisco (supported by NIGMS P41-GM103311). The volume of averaged microtubule doublets and ODA was determined by a single individual blinded to details of the case using a bounding box as shown in figure 4c. From these measurements, the percentage of the microtubule doublet that the ODA occupied was calculated (online supplementary material).

\section{Immunofluorescence}

Immunofluorescence labelling was performed as previously described [3, 21]. Imaging was conducted using a confocal microscope (SP5; Leica, Milton Keynes, UK) (online supplementary material).

\section{Results}

DNAH11 longitudinal tomograms demonstrate a loss of volume from the ODA in the proximal cilia region

In light of evidence indicating different ODA types in the proximal and distal cilium [10] (figure 1c) we created averaged longitudinal tomograms from the proximal and distal regions of the ciliary axoneme from subjects with DNAH11 mutations. Tomograms obtained from the proximal region all showed a loss of ODA volume. A representative image of a $96-\mathrm{nm}$ segment of the proximal ciliary microtubule containing four ODAs is shown in figure 2a, comparing subject \#4 who carries two frameshift loss-of-function DNAH11 alleles to a healthy control. Quantification of arm volume in four DNAH11 subjects in comparison to controls without respiratory disease $(n=3)$ shows that approximately two-thirds of the ODA volume is lost in individuals with DNAH11 defects (figure $2 \mathrm{~b})(\mathrm{p}<0.01)$. Tomograms from the remaining three DNAH11 subjects and three controls were not collected due to the technical difficulty of longitudinal electron tomography.

The retained structures seen in the proximal ODA using tomography were investigated further. In the tomogram images, it appears that a heavy chain head is retained in each ODA, presumably devoid of DNAH11 but retaining DNAH5 protein as well as the light chain proteins associated with DNAH11 such as NME8 (figure 2b). This was confirmed by immunofluorescence antibody staining from two DNAH11-deficient subjects (DNAH11 \#2 and \#7) in whom the ODA heavy chain dynein DNAH5 (ODA head component) and axonemal light chain dynein NME8 (a component predicted to be closely associated with the ODA $\beta$ heavy chain stalk) were both intact (figure $3 b$ and $d$ ).

\section{DNAH11 is localised to the proximal region of the cilium and tomograms of the distal region appear normal}

No difference was seen in longitudinal tomograms from the distal region of the cilia in subjects with three DNAH11 mutations, compared to two control subjects (figure $2 \mathrm{a}$ and b). By immunofluorescence we confirmed a proximal axonemal localisation for DNAH11 in cilia from healthy controls with no protein present in the distal cilium (figure $3 \mathrm{a}$ and $\mathrm{c}$ ).

In keeping with this finding, the ciliary beat pattern in DNAH11 subjects, as assessed by high-speed video microscopy demonstrated a higher incidence of dyskinesia in the proximal region of the cilia, compared to the distal region. In six out of seven samples there was reduced bending at the base of the cilium and preserved waveform at the tip (online supplementary video 1 and table 1) compared to healthy controls 
a) i)

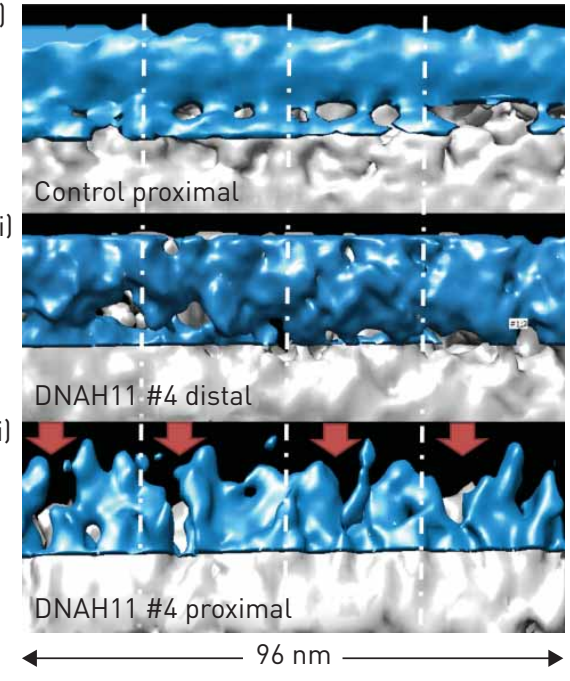

ODA

A tubule

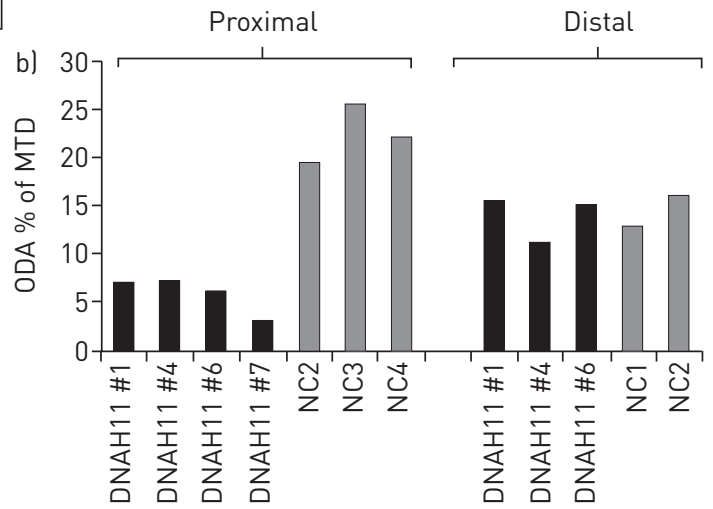

FIGURE 2 a) Representative examples of averaged, rendered and false-coloured longitudinal electron tomograms of a single $96-\mathrm{nm}$ repeating section of the cilia axoneme indicating the outer dynein arms (ODAs) (blue) and A tubule (grey): each 96-nm repeat contains four ODAs. Tomograms show four ODAs from i) the proximal axoneme of a healthy control, ii) patient \#4, who carries two frameshift DNAH11 mutations, taken from the distal region of the axoneme and iii) the proximal region. Red arrows indicate locations of the volume loss in the proximal axoneme of the DNAH11 patient. White dashed lines indicate the 24-nm repeat of each ODA. Retained structures are likely to represent DNAH5 and other light and intermediate chains of the ODA including NME8, a protein thought to be closely associated with the DNAH11 heavy chain. b) A quantification of the relative volume of the remaining structures: percentage contribution of the ODA to the microtubular doublet (MTD) volume calculated from individual averaged tomograms from nasal brushings of DNAH11 mutation primary ciliary dyskinesia patients \#1, \#4, \#6 and \#7 compared to healthy controls (NC). Data from patients \#2, \#3 and \#5 and the remaining controls could not be obtained despite multiple attempts, due to technical difficulties with the longitudinal tomography and averaging technique in plastic section. The chart shows a reduction in ODA volume in DNAH11 patients in the proximal region of the axoneme, but similar volumes between patients and controls in the distal region of the cilium.

(supplementary video 2). The incomplete beat in DNAH11 subjects results in an apparently hyperfrequent ciliary beat, as previously reported, presumably due to reduced bend and therefore a shorter effective and recovery stroke, resulting in more beats per second $[8,9]$. We speculate that this results from a lack of DNAH11 in the proximal cilium while the DNAH9- containing top (distal) half of the cilium is still moving. Taken together, high-speed video microscopy, tomography and immunofluorescence studies confirm that DNAH11 is localised to the proximal portion of the cilium, and consequently all further tomograms were taken from this region.

\section{Transverse tomograms consistently show a loss of ODA volume in DNAH11 subjects compared to controls}

Longitudinal tomograms were found to have a high rate of technical failure (14 out of 26 tomograms) and consequently could not be used routinely. Transverse cross-sectional (plane shown in figure 1c) tomograms were trialled to produce a more robust methodology. All the transverse tomograms collected $(n=19)$ contained dynein arms and could be analysed successfully. A reduction of volume of the ODA was identified in all seven DNAH11 subjects compared to healthy controls (figure 4a). The ODA proportion of the microtubular doublet had a median (interquartile range (IQR)) of $10.3 \%(9.3-10.5 \%)$ in DNAH11 subjects compared to $13.8 \%(12.9-14.4 \%)$ in healthy controls. Subjects with PCD caused by other defects (HYDIN, CCDCD65 and DRC1 mutations) had a median (IQR) ODA proportion of the microtubular doublet of $14.4 \%$ (13.1-16.0\%), similar to healthy controls. There was no overlap in the level of ODA volume loss between the DNAH11 group and either control group (figure 4a), indicating a significant loss of ODA volume arising from DNAH11 mutations $(\mathrm{p}<0.01)$. A subject with a homozygous nonsense mutation in DNAH5 (c.13285C>T; p.Arg4429*) was included as a negative control. The ODA proportion in the proximal region was $2.4 \%$, a value below the range of the DNAH11 and other control subjects.

3D image analysis showed that the volume reduction in DNAH11 subjects was from the ODA "forearm" section of the tomogram (figure $4 \mathrm{~b}$, supplementary videos 3 and 4 ). This was found consistently across all the tested subjects carrying different mutational variants within the DNAH11 gene (green annotation, figure $4 b$ ). 

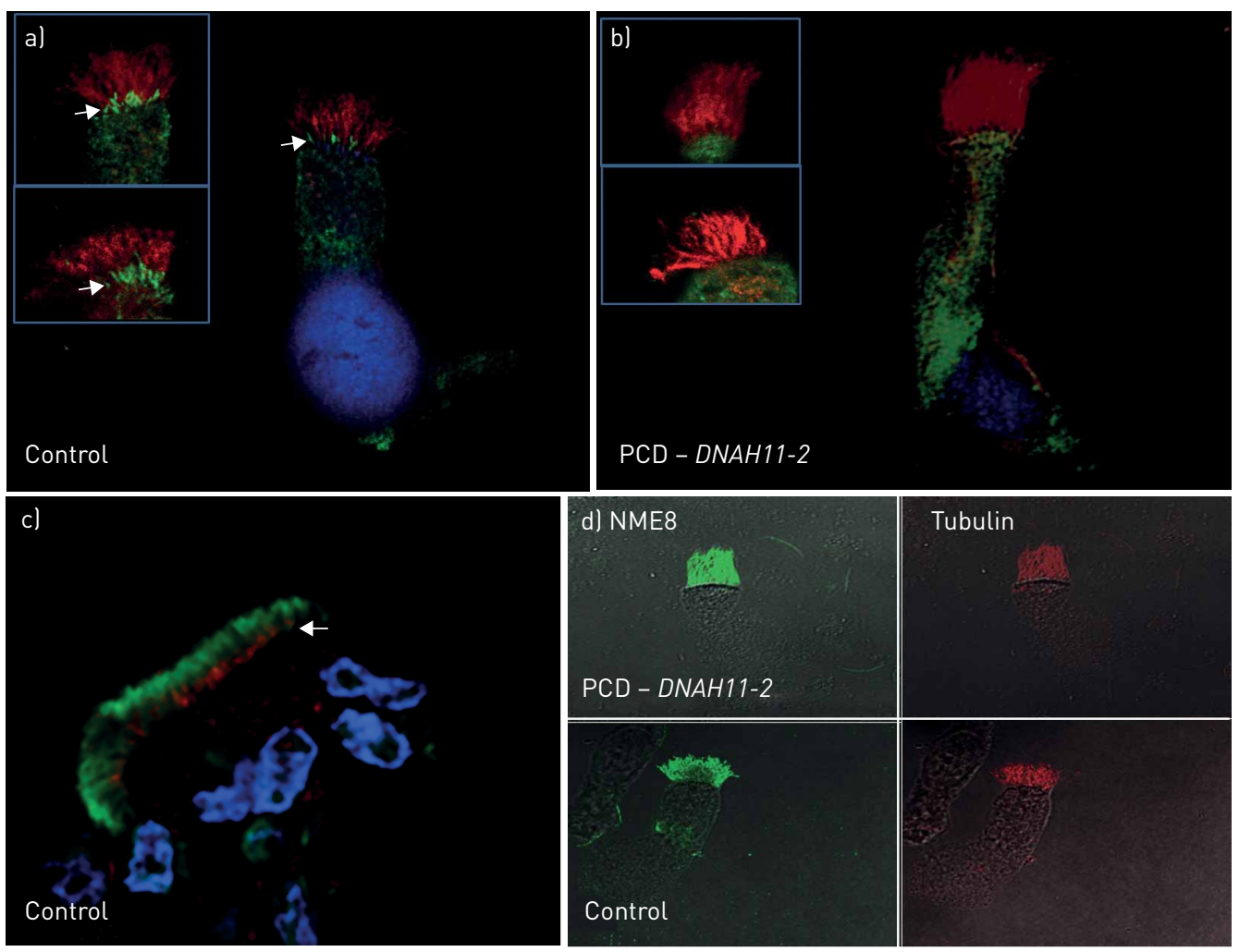

FIGURE 3 a) Immunofluoresence showing a proximal axoneme location for DNAH11 in a healthy nasal epithelial cell (green), with DNAH5 running the whole length of the cilium (red). Inset: the top of two further ciliated cells, again showing the proximal location of DNAH11 (green) with acetylated tubulin (red, top image) running the length of the cilia and DNAH9 (red, bottom image) in the distal region. White arrows indicate the position of DNAH11 throughout. b) High-resolution immunofluorescence from patient \#2 who carries a frameshift and premature truncation mutation in the gene. DNAH11 (green) is absent from the cilia. Whereas DNAH5 (main image), acetylated $\alpha$-tubulin (top inset) and DNAH9 (bottom inset) are present throughout the length of the cilia (shown in red). c) An alternative antibody to DNAH11 (red) was used to validate results (Abcam ab126571) and to show presence of DNAH11 in the peripheral airways. Cilia are marked in green with acetylated tubulin. Ciliated epithelium was obtained from a lung resection. d) Cilia are marked by acetylated $\alpha$-tubulin antibody (red); NME8, a protein predicted to be closely associated with the $\beta$-dynein heavy chain, is shown to be retained (green) in an individual with primary ciliary dyskinesia (PCD).

\section{Discussion}

We show that DNAH11 mutations result in a characteristic abnormality of the ciliary ultrastructure detectable by electron tomography, but not traditional TEM. The defect is specific to the "forearm" of the ODA and is detected only at the proximal portion of the cilium where DNAH11 is located.

The transverse section electron tomography technique used in this study is advantageous in that it uses existing fixed embedded samples already stored for diagnosis by standard TEM, thereby avoiding additional sampling. A strength of the technique used in this study is the quantification of results and the use of the MTD volume as an internal control during quantification, to minimise bias introduced by image analysis, such as manual selection of image features and thresholding. Quantification showed no overlap in ODA volume measurements between healthy or PCD controls and DNAH11 subjects, confirming the clear-cut nature of the underlying defect at this imaging resolution. The reliability of the test results suggests that the technique has potential use in the diagnostic pathway for highly atypical PCD cases.

Using immunofluorescence and electron tomography we demonstrate that DNAH11 mutations do not result in loss of the entire ODA. We found that a partial heavy chain of the ODA head region (presumed to be the DNAH5 protein), along with at least part of the remaining ODA structure (NME8-positive), remains intact along the length of the proximal cilia region in patients with DNAH11 mutations. The localisation of these retained structures, coupled with the proximal nature of the DNAH11 loss, provides the likely explanation for the longstanding puzzle of apparently normal cilia structure in DNAH11 patients when using conventional TEM. Figure 5 shows a cartoon to demonstrate the predicted model of the ODA 
b) i)

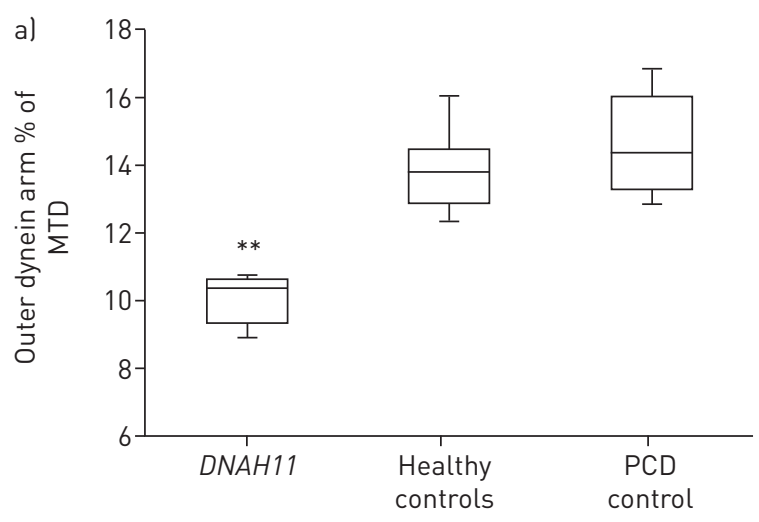

c) i)

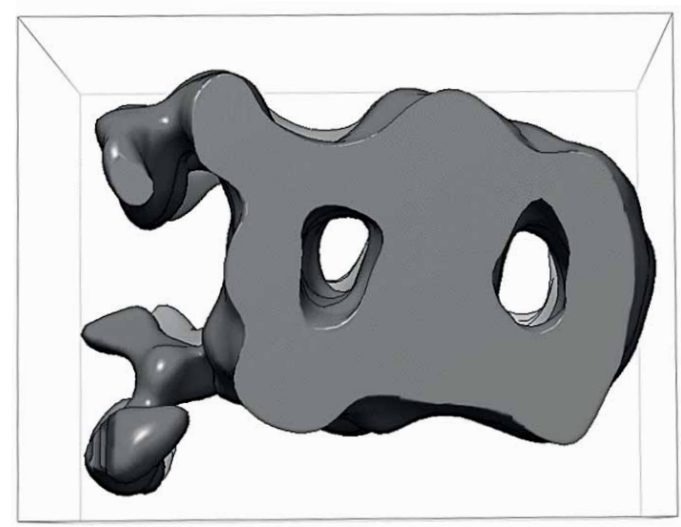

ii)
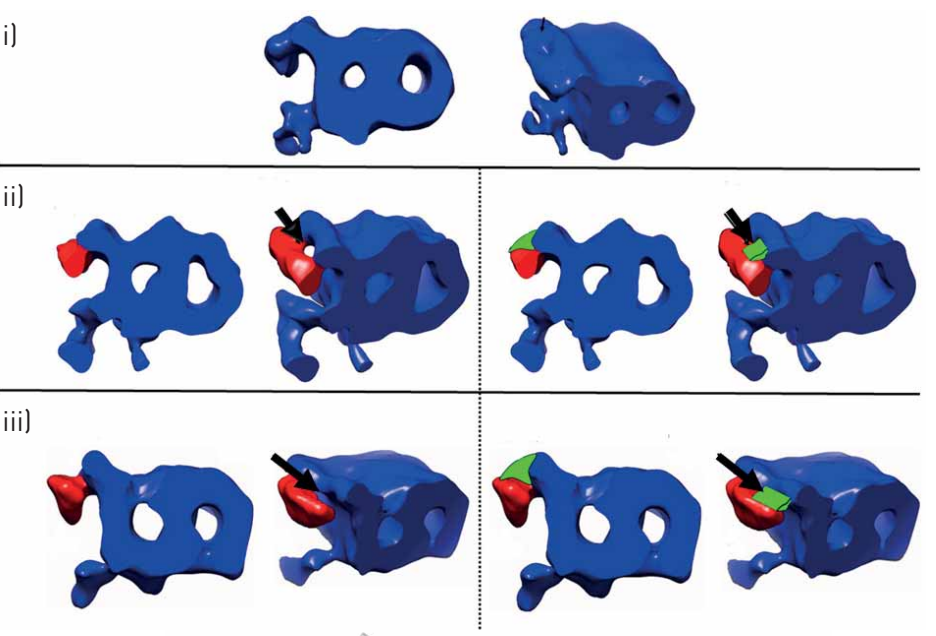

ii)

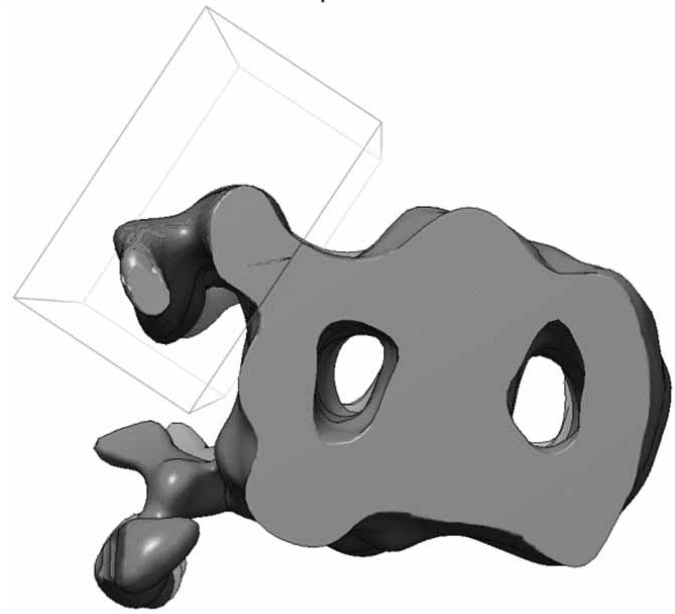

FIGURE 4 a) Data from volume measurements from averaged transverse tomograms from the proximal portion of the axoneme from six healthy controls, seven DNAH11 mutation patients and six subjects with primary ciliary dyskinesia (PCD) due to other defects (HYDIN $\mathrm{n}=2, C C D C 65 \mathrm{n}=3$ and $D R C 1 \mathrm{n}=1$ ). There is a significant difference between the DNAH11 mutation group and the two control groups. ${ }^{* *}$ : $\mathrm{p}<0.01$ (Mann-Whitney U-test). b) Representative transverse section tomograms averaged, rendered and false-coloured, showing two different angles for each case: i) the tomographic average of a microtubular doublet (MTD) (blue), from a healthy control; ii) tomographic averages from DNAH11 mutation patient \#1, who carries a premature truncation mutation and frameshift mutation; iii) tomographic averages from DNAH11 mutation patient \#2, who carries a different premature truncation mutation and frameshift mutation. The predicted location of DNAH5 is coloured red; the green triangles have been added to the images on the right to indicate where DNAH11 is predicted to be missing. c) Tomograms from a healthy control as visualised in Chimera showing the bounding box method for quantification of i) the MTDs and ii) the outer dynein arm (ODA), enabling the calculation of the percentage volume of the ODA out of the total MTD volume (used in a)).

loss in DNAH11 cases. These data arising from 3D tomographic imaging advance our understanding of the cell biology of PCD disease and how it affects the cilia, such that the majority of structures remain completely undisturbed while a highly specific loss of only a portion of the ODA is sufficient to cause ineffective mucociliary clearance and its clinical consequences.

In this study we have shown that transverse section electron tomography can differentiate between DNAH11 and control cases without the need for more complex longitudinal section or cryo-electron tomography techniques. The proximal location of DNAH11 means appropriate selection of a proximal area for transverse electron tomography is crucial for this technique. In the present study the presence of microvilli was used as a marker for proximity to the cell surface and therefore the DNAH11 region; however, this method is imperfect, as occasional tip structures from bent cilia could reside in this region. The clear distinction between control and DNAH11 cases suggest this was not a major limitation in defining a DNAH11 defect in the present study. Other limiting factors of the electron tomography technique is that it is time-consuming, labour intensive and requires specialist training. It can take days to collect, reconstruct and average each tomogram, resulting in only a small number of cross-sections being assessed and precluding its use as a high-throughput diagnostic screening tool. However, high throughput was never our intention, and this technique could be reserved for specialist cases at a small number of 
a) i

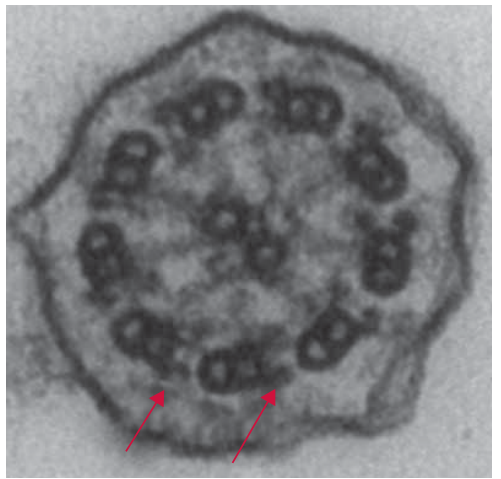

b) i) Inner arm

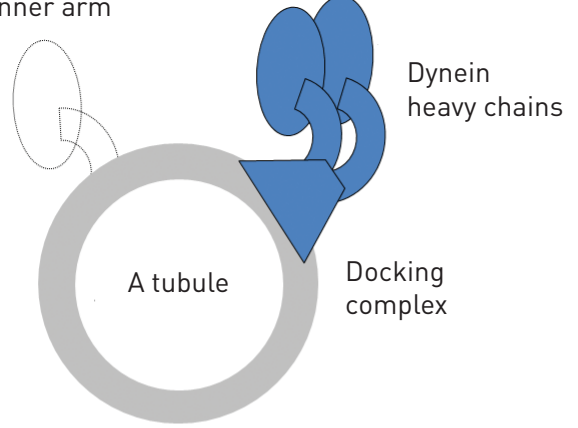

c) i)

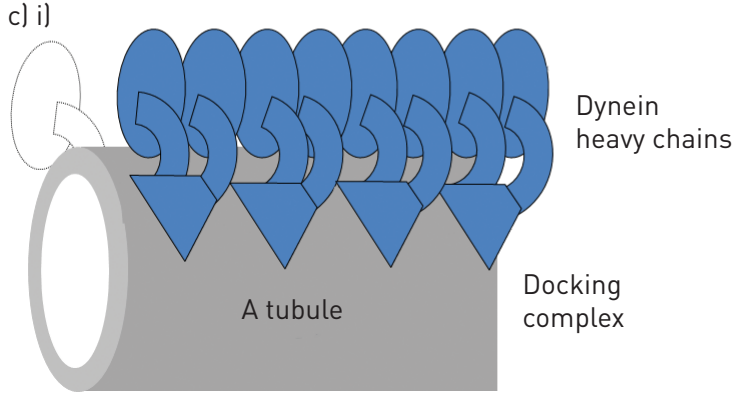

ii)

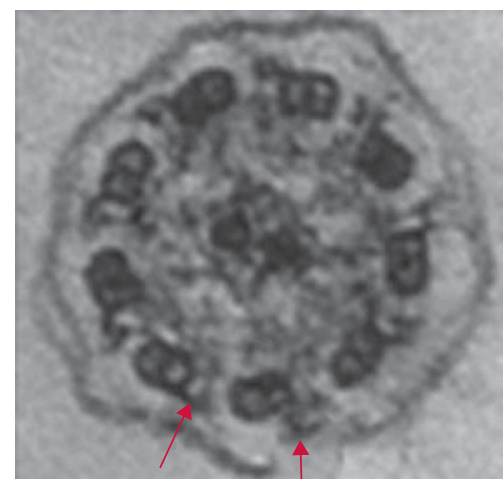

ii)

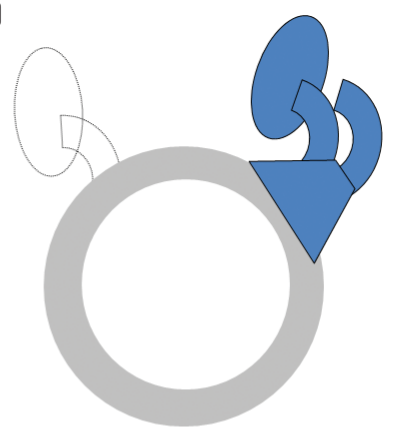

ii)

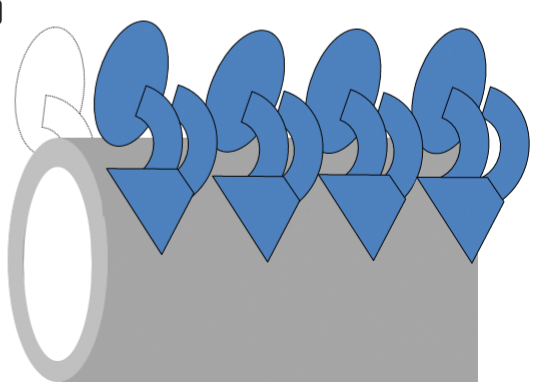

FIGURE 5 a) Electron micrographs showing normal cilia ultrastructure in nasal brushings from i) a healthy control and ii) DNAH11 mutation patient \#1. Simplified models of the expected appearance of a DNAH11 defect in b) transverse section and c) longitudinal section, shown for i) a healthy control and ii) a DNAH11 mutation. Blue: outer dynein arm and its docking complex; white: inner dynein arm; grey: the A tubule of the microtubular doublet.

reference centres since specimens which have already been embedded can easily be transported between centres. Use of chemically embedded sections and the restricted tomographic tilt range $\left( \pm 65^{\circ}\right.$ rather than $\pm 90^{\circ}$ ) are both factors that limit the full potential of tomographic resolution. The majority of the DNAH11 protein volume when imaging the ODAs in transverse section is in the depth (z-direction), which results in loss of resolution compared to image acquisition in the longitudinal plane. Use of longitudinal sections or cryo-electron tomography would overcome some of the resolution limitations and could thereby further elucidate the structural role of human DNAH11 [20]. However, we found longitudinal tomography technically challenging and were not able to collect a full dataset using this technique (figure 2b). Cryo-electron tomography is not practical for diagnostic evaluation in clinic or for the review of existing, previously unsolved, clinical samples, due to the requirement for a cryo-electron microscope and the need for immediate preparation of samples following biopsy or culture [12].

Immunofluorescence can be used for confirmation of a DNAH11 defect. However, antibodies for DNAH11 are not widely available and others have shown that the protein remains present in cilia from individuals with PCD caused by pathogenic DNAH11 missense mutations since the staining is indistinguishable from controls [10]. One individual (subject \#5) in the present study carries a homozygous missense mutation. Cilia from this subject demonstrate the same $25 \%$ tomographic volume loss as seen in the other six subjects carrying predicted null mutations (premature truncation and 
frameshift mutations). We speculate that the ability to detect protein loss arising from missense mutations by electron tomography is due to the superior resolution of the technique compared to immunofluorescence; however, this would need to be verified in further missense variants. It is possible that the difference in ODA volume could represent a conformational change in the dynein rather than a direct loss of the DNAH11 protein. This would provide another possible explanation for loss of volume in a missense mutation, where some protein is predicted to remain present. Interestingly, DougherTy et al. [10] and data in this study have shown that DNAH9 can enter the proximal area of the axoneme in the absence of DNAH11. Our study suggests that any such replacement protein still does not fully occupy the structural defect left by DNAH11 protein.

Therefore, this study provides proof of concept that electron tomography can be used to confirm a diagnosis of PCD, where traditional TEM appears normal. We suggest that electron tomography, due to the requirement for microscopy equipment and its time-consuming nature, could be used in specialist centres in cases where other tests for PCD have been inconclusive and the genetic testing if available rests upon variants of uncertain significance that require functional confirmation (e.g. unpublished missense mutations). The online supplementary material demonstrates an example case study where tomography was used to confirm a diagnosis in a patient with a missense variant in DNAH11.

In summary, we have shown a consistent ultrastructural defect in subjects that carry deleterious DNAH11 mutations once thought to have "normal ultrastructure". We have shown that the tomography technique has good resolution and accuracy for the detection of PCD defects in DNAH11-deficient patients with apparently normal ultrastructure. Cilia electron microscopy has facilitated the major advances in PCD. Improved resolution and 3D visualisation of tomography has promise to provide important cell biological insights into the causes and variability of PCD along with an improved understanding of the significance of novel gene mutations.

\section{Acknowledgements}

We would like to thank the PCD families for their participation in this study and NHS England for support of the UK PCD specialised service. We would like to acknowledge the work of Michael Simpson (King's College London, London, UK), Lucy Jenkins and Christopher Boustred (NE Thames Regional Molecular Genetics Service at Great Ormond Street Hospital for Children NHS Foundation Trust, London) for genetic analysis. We acknowledge Wellcome Trust award WT091310 to UK10K and are grateful to the UK10K consortium (investigators listed at www.uk10k.org/), in particular the Rare Diseases Group, for whole-exome sequencing of PCD patients. Authors are members of the BESTcilia FP7 and COST Action BEAT-PCD (BM1407).

\section{References}

1 Lucas JS, Burgess A, Mitchison HM, et al. Diagnosis and management of primary ciliary dyskinesia. Arch Dis Child 2014; 99: 850-856.

2 Lucas JS, Barbato A, Collins SA, et al. European Respiratory Society guidelines for the diagnosis of primary ciliary dyskinesia. Eur Respir J 2017; 49: 1601090.

3 Shoemark A, Frost E, Dixon M, et al. Accuracy of immunofluorescence in the diagnosis of primary ciliary dyskinesia. Am J Respir Crit Care Med 2017; 196: 94-101.

4 Shoemark A, Dixon M, Corrin B, et al. Twenty-year review of quantitative transmission electron microscopy for the diagnosis of primary ciliary dyskinesia. J Clin Pathol 2012; 65: 267-271.

5 Greenstone MA, Dewar A, Cole PJ. Ciliary dyskinesia with normal ultrastructure. Thorax 1983; 38: 875-876.

6 Knowles MR, Daniels LA, Davis SD, et al. Primary ciliary dyskinesia. Recent advances in diagnostics, genetics, and characterization of clinical disease. Am J Respir Crit Care Med 2013; 188: 913-922.

7 Boon M, Smits A, Cuppens H, et al. Primary ciliary dyskinesia: critical evaluation of clinical symptoms and diagnosis in patients with normal and abnormal ultrastructure. Orphanet J Rare Dis 2014; 9: 11.

8 Knowles MR, Leigh MW, Carson JL, et al. Mutations of DNAH11 in patients with primary ciliary dyskinesia with normal ciliary ultrastructure. Thorax 2012; 67: 433-441.

9 Schwabe GC, Hoffmann K, Loges NT, et al. Primary ciliary dyskinesia associated with normal axoneme ultrastructure is caused by DNAH11 mutations. Hum Mutat 2008; 29: 289-298.

10 Dougherty GW, Loges NT, Klinkenbusch JA, et al. DNAH11 localization in the proximal region of respiratory cilia defines distinct outer dynein arm complexes. Am J Respir Cell Mol Biol 2016; 55: 213-224.

11 Richards S, Aziz N, Bale S, et al. Standards and guidelines for the interpretation of sequence variants: a joint consensus recommendation of the American College of Medical Genetics and Genomics and the Association for Molecular Pathology. Genet Med 2015; 17: 405-424.

12 Lin J, Yin W, Smith MC, et al. Cryo-electron tomography reveals ciliary defects underlying human RSPH1 primary ciliary dyskinesia. Nat Commun 2014; 5: 5727.

13 Burgoyne T, Dixon M, Luther P, et al. Generation of a three-dimensional ultrastructural model of human respiratory cilia. Am J Respir Cell Mol Biol 2012; 47: 800-806.

14 Olbrich H, Schmidts M, Werner C, et al. Recessive HYDIN mutations cause primary ciliary dyskinesia without randomization of left-right body asymmetry. Am J Hum Genet 2012; 91: 672-684.

15 Horani A, Brody SL, Ferkol TW, et al. CCDC65 mutation causes primary ciliary dyskinesia with normal ultrastructure and hyperkinetic cilia. PLoS One 2013; 8: e72299.

16 Wirschell $\mathrm{M}$, Olbrich $\mathrm{H}$, Werner $\mathrm{C}$, et al. The nexin-dynein regulatory complex subunit DRC1 is essential for motile cilia function in algae and humans. Nat Genet 2013; 45: 262-268. 
17 Austin-Tse C, Halbritter J, Zariwala MA, et al. Zebrafish ciliopathy screen plus human mutational analysis identifies C21orf59 and CCDC65 defects as causing primary ciliary dyskinesia. Am J Hum Genet 2013; 93: 672-686.

18 Kremer JR, Mastronarde DN, McIntosh JR. Computer visualization of three-dimensional image data using IMOD. J Struct Biol 1996; 116: 71-76.

19 Mastronarde DN. Dual-axis tomography: an approach with alignment methods that preserve resolution. J Struct Biol 1997; 120: 343-352.

20 Nicastro D, Schwartz C, Pierson J, et al. The molecular architecture of axonemes revealed by cryoelectron tomography. Science 2006; 313: 944-948.

21 Frommer A, Hjeij R, Loges NT, et al. Immunofluorescence analysis and diagnosis of primary ciliary dyskinesia with radial spoke defects. Am J Respir Cell Mol Biol 2015; 53: 563-573. 